\title{
La formación metodológica y el éxito gerencial
}

\author{
Romero Salazar, Juan José ${ }^{\star}$
}

\section{Resumen}

En este trabajo se describe el enfoque epistemológico del programa de metodología de la investigación aplicado en la Maestría en Gerencia de la Universidad Nacional Experimental de Guayana, a partir del año 2001. Los objetivos son, promover la reflexión crítica acerca de los modelos dominantes en la formación gerencial, especialmente los vinculados con el enfoque empíricoanalítico e incorporar la perspectiva de la diversidad metodológica, para situar, con suficiente reat ismo, el ejercicio de la dirección como una acción humana de gran complejidad. La metodología utilizada para elaborar este trabajo está enmarcada en los lineamientos de la investigación-acciónparticipante. En este sentido, el autor, como sujeto social, al lado de los cursantes de la maestría, describen una acción social centrada en el compromiso político-académico para la transformación de la gerencia de corte instrumental. Como resultado de la aplicación de esta metodología se diseñaron los principales componentes del modelo psicosocial de la gerencia y se concluyó en la necesidad de construir su viabilidad, al lado del compromiso con el proyecto científico nacional.

Palabras clave: Formación metodológica, éxito gerencial, modelos de investigación, compromiso social.

\section{Methodological Formation and Management Success}

\section{Abstract}

This paper describes the epistemological approach in the research methodology program offered in the Masters Degree Program in Management in the National Experimental University of Guyana beginning in 2001. The objectives are to promote critical thought in reference to the dominant models used in management programs, and specially those related to the empiric-analytical focus.

Recibido: 01-11-07 . Aceptado: 02-04-23

Sociólogo, Magíster en Gestión Universitaria. Profesor de Metodología de la Investigación. Adscrito a la Coordinación General de Investigación y Postgrado de la Universidad Nacional Experimental de Guayana (UNEG), Puerto Ordaz. Venezuela. Telf: (58-286) 9618324.

E-mail: juanjose-r-s@cantv.net 
Also it is necessary to incorporate the perspective of methodological diversity in order to focus the exercise, with sufficient realism, in the direction of highly complex human action. The methodology used to elaborate this paper is framed within lines of research-action-participation. In this vein, the author, as a social subject accompanying the participants in the Masters program, describes social action as centered on the political-academic commitment in order to transform instrumental management. As a result of the application of this methodology, the principal components of the psycho-social model of management are designed, and the conclusion focuses on the need to construct its viability, in accord with the commitment to support the national scientific project.

Key words: Methodological formation, management success, research models, social commitment.

\section{Introducción}

La formación metodológica para el desarrollo de una Gerencia Nacional Alternativa, surge como una opción imprescindible para superar el pensamiento único neoliberal que, en gran medida ha condicionado a los directivos de las empresas del Estado Venezolano en los últimas décadas, mermando el compromiso que les corresponde asumir con lo público. De ello deriva la exigencia de producir un nuevo modelo de enseñanza, que acompañe los cambios significativos, que se están produciendo en las relaciones laborales del mundo actual, especialmente en Venezuela, donde las modificaciones del modelo sociopolítico con un nuevo texto constitucional, a partir del año 2000, requiere de un sistema científico a nivel de la formación de los directivos de las empresas públicas, para elevar las competencias profesionales y profundizar los valores patrióticos de los lideres comprometidos con la gobernabilidad del país.

En Venezuela se está produciendo un cambio cuyo fundamento doctrinario tiene en el pueblo a su sujeto histórico, integrado en sociedad política y so- ciedad civil. En estas condiciones de transformación, se ha venido planteando para el país un desarrollo económico intensivo, un acelerado avance científico tecnológico y una actualización de su modelo de planificación nacional, lo cual amerita una sistemática preparación política y profesional de los gerentes, pues se trata de la revalorización de la función pública. Por ello, el programa aplicado por el autor en la Maestría en Gerencia de La Universidad Nacional Experimental de Guayana, en Venezuela, puede considerarse como parte integrante de un sistema más amplio de formación y desarrollo de los cuadros dirigentes del Estado, en correspondencia con las transformaciones que se llevan a cabo en la Gerencia Pública Nacional.

A tal efecto, el Programa de formación metodológica para el éxito gerencial, que se presenta en este trabajo, coloca el énfasis en el análisis crítico del enfoques empírico-analítico, que prácticamente opera como pensamiento único en las llamadas Escuelas de Gerencia. Así mismo, evalúa la situación actual de la formación metodológica y su grado de correspondencia con el proceso de transformación a desarrollar en la administración pú- 
blica Venezolana. Luego, presenta una propuesta académica para la formación y desarrollo de los aspirantes al nivel de Maestría en Gerencia, con el propósito de que participen, como actores del proceso de cambio socio-político que vive el país. En realidad, se trata de un fortalecimiento educativo de los directivos para una gerencia científica, identificada con los criterios de equidad y justicia social, que pasan a ser la mayor prioridad de la nueva República, para alcanzar el desarrollo sostenible, lo cual requiere así mismo, de un sistema de formación y desarrollo, que contemple la capacitación técnica y la formación de valores de los dirigentes,

La propuesta de formación metodológica para los gerentes aspira que éstos, a través de la investigación adquieran una adecuada responsabilidad social. De allí que la connotación del éxito, que aquí se maneja, no está, de ningún modo relacionada con el logro exclusivo de los beneficios económicos y el ascenso en la estructura social de la elite, tal como lo postula el modelo burocrático, que se analiza en este texto. Más bien, el verdadero significado del éxito que postula este programa de formación metodológica, fundamentalmente tiene que ver con la apropiación de conocimientos avanzados, para dotar al país de lideres con perfil propio, preparados profesionalmente, con habilidades técnicas y valores espirituales para enfrentar la violenta mundialización dominada por la lógica del programa neoliberal. Puntualmente, la importancia del programa de metodología gerencial está representada por la disposición de aportar los conocimiento y las herramientas para el trabajo del direc- tivo Venezolano, en el ejercicio de sus tres funciones básicas, a saber: Función de líder político, función de dirigente social y función de gerente ejecutivo.

Por lo tanto, la labor de la escuela de gobierno está orientada a lograr un perfil polivalente, en la formación integral del cuadro políticamente comprometido, sensibilizado socialmente y gerencialmente preparado para la gobernabilidad de nuestro país.

En cuanto a la importancia práctica del trabajo, tiene que ver con el hecho de haber detectado que las deficiencias de cuadros para dirigir el nuevo proceso sociopolítico Venezolano, se deben básicamente a la inexistencia de una instancia organizada para coordinar la preparación y elevación de la calificación de los cuadros gerenciales en funciones de gobierno. Así mismo se comprobó que los intentos para diseñar planes y programas, en este campo han sido aislados. Por esta razón, resulta de vital importancia este sistema de formación y desarrollo, para crear una red metodológica que integre algunas experiencias de formación de los directivos y atender la creación de una reserva estratégica para el relevo de los actuales dirigentes.

En razón de lo antes expuesto, para la implantación del sistema de formación y desarrollo de los cuadros directivos, se creará, como una innovación académica, una escuela de gobierno, que se convertirá en la instancia organizada, para dirigir con éxito esta fase de transición política y económica, e impulsar los cambios que impone la Constitución Bolivariana, cuyo paradigma central es el ejercicio de la democracia partici- 
pativa y protagónica del pueblo, considerado éste como el eje del desarrollo sustentable.

\section{Direccionalidad de la metodológica gerencial}

La importancia de los programas de metodología de la investigación, constituye un lugar común para el avance del proceso de producción de conocimiento. Su relevancia está en todas las áreas del saber humano, tanto en la relación del hombre con la naturaleza, como en el vínculo entre ciencia y sociedad, así como también en el desarrollo de la educación y la cultura, y en la administración y gerencia. Ello revaloriza el papel del metodólogo, que en este sentido, pasa a ser el actor principal en la confección de las cada vez más proliferas líneas de investigación. Quienes asumen la formación metodológica, han de estar conscientes, que este es un ejercicio organizado y sistemático que apunta hacia una heterogeneidad temática difícil de inventariar en toda su creciente amplitud. En el terreno epistemológico se considera que la metodología de la investigación es una disciplina que discute, por una parte, los problemas teóricos del método científico, y por la otra, trabaja las metódicas operacionales de la práctica científica.

En la Universidad Nacional Experimental de Guayana, desde el primer trimestre del año 2001 se diseñó y se empezó a aplicar el programa de metodología de la investigación, dirigido a los cursantes de las maestrías en gerencia de recursos humanos, gerencia de finanzas y gerencia de la producción. El objetivo de este curso consiste en dotar al maes- trante de los conocimientos teóricos-metodológicos para la elaboración de la tesis de grado, a partir de la formulación de un objeto de estudio, considerado como una acción social sometida a rastreamiento y pesquisa, a partir del campo de conocimiento del investigador. Por ello se considera al aspirante a magíster en gerencia como el sujeto activo del proceso, que convertido en agente social tiende a problematizar su interés cognoscitivo, a través de tres preguntas directrices: ¿Qué investigar? ¿Cómo investigar? ¿Para que investigar? En principio, las respuestas a estas interrogantes les ha permitido asumir dos niveles epistemológicos, uno relativo a la dimensión paradigmática de lo teórico-metodológico, y otro a la postura ética y cultural, que les corresponde asumir como investigador.

En este marco se han trabajado algunos de los obstáculos epistemológicos, Bachellardianos. Esto permitió a los integrantes del curso, cuestionar, en primer lugar, la postura precientífica, que limita la práctica investigativa a la experiencia básica: "En la formación de un espíritu científico, el primer obstáculo es la experiencia básica, es la experiencia colocada por delante y por encima de la crítica, que, ésta si, es necesariamente un elemento integrante del espíritu científico" (Bachellard, 1976: 27). También, se advirtió acerca del papel que juega el conocimiento general como otro de los obstáculos en la formación del espíritu científico, en términos de que: "...esta ciencia de lo general, es siempre una detención de la experiencia, un fracaso del empirismo inventivo. Conocer el fenómeno general, prevalerse de ello para comprender todo [...] Hay en efecto un goce inte- 
lectual peligroso en una generalización precoz y fácil" (Bachellard, 1976:66). Pero además de esta función generalizante, se advirtió acerca del conocimiento unitario y pragmático como obstáculo para el conocimiento, por cuanto plantea la unidad y poder de la naturaleza, a partir de una postura precientífica que atribuye como "coeficiente de realidad" a todo lo que es natural: "...todas las dificultades se resuelven ante una visión general del mundo, mediante una simple referencia a un principio general de la Naturaleza. [...] la idea de una Naturaleza homogénea, armónica, tutelar anula todas las singularidades, todas las contradicciones, todas las hostilidades a la experiencia" (Bachellard, 1976: 99).

En este programa de metodología para los aspirantes al master de gerencia, se desarrolla el debate acerca de la objetividad científica, a la luz del "triunfo del espíritu científico sobre los distintos obstáculos epistemológicos". La objetividad se plantea como una tendencia que el investigador decide transitar con realismo, para sustituir el ingenuo optimismo tecnocientífico, y la insegura expectativa de alcanzar automáticamente los productos de una ciencia objetiva y benefactora, los cuales resultan falaces. De allí resulta ineludible, seguir a Bachellard, al considerar que el objeto no puede concebirse de inmediato como objetivo, y aceptar que hay que establecer una ruptura entre el conocimiento sensible y el conocimiento científico, ya que el conocimiento sensible está mediatizado por el "pragmatismo y el realismo inmediatos", lo cual determina un "falso punto de partida y una falsa dirección". Desde esta perspectiva, el leit-motiv de la enseñanza metodológi- ca gerencial, ha consistido y consistirá en mostrar que el proceso investigativo tiene un carácter marcadamente social, y que el deber intelectual del gerente es siempre evidenciar la inserción, que como investigador le corresponde, dentro de la estructura social objeto de estudio, es decir, de la totalidad de la cual forma parte. La investigación gerencial, como proceso social, establece una relación de identidad sujeto-objeto, en tanto que la categoría gerencia es una acción social, desarrollada por el propio gerente en un ámbito espacial y en una temporalidad históricamente determinada.

\section{Discusión epistemológica de la diversidad}

En Venezuela, la discusión epistemológica, debe abrirse para superar la unilateralidad, que predomina en la enseñanza institucionalizada mediante el modelo rígido que se ha incorporado en los programas de estudio de las Universidades, los cuales contienen una absolutización del método empírico-analítico como único método de la investigación científica, que se adecua para todas las disciplinas, convirtiéndose en el metaparadigma, que con suficiente amplitud conceptualiza Damiani: "Entendemos como metodología empírico-analítica aquella concepción de la metodología que caracteriza al objeto de investigación como susceptible de ser disgregado en sus partes componentes [...] en efecto, es concebida como constitutiva de una pretendida "ciencia normal" nunca sujeta a crítica, perteneciente al ámbito de las verdades autoevidentes compartidas por la comunidad de los científicos" (Damiani, 
1994:12). Este autor principalmente le cuestiona, a la metodología empíricoanalítica, el sesgo hacia el instrumentalismo metodológico que privilegia procedimientos operativos por encima de la reflexión metodológica. Así evidencia la intencionalidad de naturaleza positivista al presentar la actividad científica como independiente, fundada en principios autónomos y neutrales de la objetividad. Esta convicción lleva a Damiani a un juicio terminante, cuando sostiene que ninguna metodología puede autoconvalidarse, y su éxito tiende a justificarse en base a la posición filosófica del investigador. Por tal razón, le resulta obligado asumir la diversidad metodológica, según la cual el oficio del investigador se despliega en una interacción compleja, determinada por condiciones materiales y culturales. De esta manera, el investigador debe contextualizar el proceso investigativo, pues no le es posible desprenderse de la postura epistemológica, que le es inmanente en el plano de la realidad de la que forma parte.

De acuerdo con el planteamiento de Tomas Kuhn (1981), en las comunidades científicas han proliferado distintas modalidades de investigación, y en las ciencias sociales las posibilidades de abordar el objeto de estudio, para producir conocimientos son infinitas. Ante esta realidad toma fuerza la tesis de la diversidad metodológica para la flexibilización de estrategias y enfoques cognoscitivos. Ya la diversidad es imperativa, para hacer frente al monopolio institucional que se ha creado alrededor del modelo empírico-analítico. En el plano de la formación gerencial, este modelo tiene una expresión ideológica en: "...la idea de la geren- cia, como una multidisciplina desprovista de condicionamientos sociales y como una entidad autónoma, que soslaya el carácter epistemológico de la administración como ciencia de la dirección. Muy por el contrario, la elite empresarial ha propagado la tesis de la neutralidad valorativa, cuya intencionalidad está conscientemente orientada a fortalecer la estructura de poder en la empresa capitalista" (Romero, 2000:11). Esta concepción de gerencia está vinculada con la matriz epistemológica del estructural- funcionalismo y entraña una idea de la dirección, basada en la lógica de la dominación ideológica, en tanto que refuerza los mecanismos de alineación.

Ante el predominio de la racionalidad instrumental de la gerencia capitalista, es condición sine qua non, la tesis de la diversidad metodológica, para la conformación de verdaderas comunidades científicas en los términos de kuhn (1981), y la preparación integral de los investigadores, a través del ejercicio de la multiplicidad de prácticas científicas que tienen cabida en las ciencias sociales. Sin embargo, en Venezuela los modelos alternativos no han sido incorporados abiertamente a los programas de metodología en la formación gerencial, donde impera el pragmatismo de la tecnoburocracia, que se ha conformado como clase emergente con las elites que controlan la producción de las grandes empresas e "inciden en el aparato del Estado para fundamentar el ejercicio del poder político" (Poulantzas, 1977). A pesar de los esfuerzos de algunas instituciones académicas, donde se estudia la gerencia pública, no ha sido posible incorporar cualitativamente un pensamiento humano y 
una práctica directiva de corte social, por cuanto este es uno de los campos de mayor influencia de la policy making.

\section{Modelos clásicos en investigación social}

Entre las alternativas que se pueden describir, en un esquema de diversidad metodológica, están la sociología de la comprensión y el método dialéctico, que incorporan desde distintas perspectivas, el concepto de totalidad, en el análisis de la realidad social. En esta vertiente de investigación alternativa, al modelo empírico-analítico, se destacan Marx y Weber como los representantes clásicos. Sus concepciones y tesis han contribuido a la pluralidad del pensamiento sociológico y por consiguiente al ejercicio de diferentes y opuestas praxis científicas, que se contextualizan, a través de una redefinición del objeto y método para el análisis científico.

Los planteamientos de Weber constituyen la base de la matriz epistemológica de la fenomenología y etnografía. Esta matriz epistemológica tiene su soporte fundamental en la dimensión subjetiva del accionar social, que considera que la estructura de la sociedad no es la que determina el accionar individual, pues ella, la estructura social, está anticipadamente orientada por el comportamiento del accionar individual y es siempre el resultado de éste.

Weber, en la propuesta de la sociología comprensiva, incorpora la interpretación del sentido de una acción para establecer un vinculo indisoluble entre explicación y comprensión: "En resumidas cuentas, el objeto de la sociología de la comprensión es la acción humana en cuanto referida a la orientación de la acción de otros individuos, determinada por esta referencia e interpretable en virtud del "sentido subjetivamente intencionado" del actor que la efectúa. Lejos de contraponerse, el proceso de compresión y el de explicación coinciden" (Damiani, 1994:153). Realmente, Weber se centra en la racionalidad de la acción humana, como modelo ideal. A partir de allí, interpreta las distintas conductas sociales, y convierte la categoría de los tipos ideales en el fundamento del proceso de producción de conocimiento. Dicho de otra manera, en la relación entre la teoría y la realidad, los tipos ideales constituyen el elemento fundante y se convierten en la esencia de su proposición metodológica. En el modelo Weberiano, la investigación plantea exigencias científicas que incluyen la confirmación de hipótesis formuladas para validar el conocimiento. Ello se ejecuta a través de "hipótesis interpretativas" que es necesario someter a verificación empírica para una comprensión legitima de la realidad y para superar el inmediatismo, o más bien, "el entender inmediato". En este sentido, esta propuesta metodológica asume el análisis histórico social, que ha sido la más clara intencionalidad de su autor: "...las construcciones típico-ideales del desarrollo y la historia son dos cosas a las que es preciso distinguir nítidamente, y que la construcción ha sido aquí solamente el medio de cumplir la imputación válida, sistemáticamente, de un proceso histórico a sus causas reales, dentro del circulo de las posibles de acuerdo con el estado de nuestros conocimientos" (Weber ,1973:91). 
Desde una posición opuesta se plantea el análisis marxista en la teoría crítica de la sociedad, en la cual se privilegia el carácter contradictorio de la realidad social. Esta se caracteriza como una totalidad compleja de múltiples determinaciones, donde el objeto de la actividad científica consiste en la apropiación conceptual de las relaciones sociales, incorporadas a un proceso de permanente transformación, producto de las contradicciones que se generan en el seno de esas relaciones. Este enfoque dialéctico está orientado a la investigación de lo social y filosóficamente enfatiza la historicidad de los fenómenos, destacando el movimiento contradictorio en la totalidad social de la que forma parte, así lo plantea Marx (1979) en la Miseria de la Filosofía: "Las categorías económicas no son más que las expresiones teóricas, las abstracciones de las relaciones sociales de producción" Relaciones en las que se generan conflictos, que derivan de la oposición que las clases sociales mantienen. Estas contradicciones tienen un carácter marcadamente económico y social, por cuanto las clases sociales mantienen posiciones diferentes en la producción de bienes, y con relación a la propiedad privada sobre los medios de producción. En el Manifiesto Comunista Marx y Engels (1974) expresan: "La historia de las sociedades hasta ahora existidas es la historia de la lucha de clases"

Lo que se puede considerar como enfoque epistemológico Marxista, está centrado en la relación dialéctica que acontece en la producción de conocimiento entre la práctica y la teoría. Este enfoque establece que el criterio de validez científica se logra en la propia prácti- ca, lo que implica una constatación permanente de la teoría con la realidad concreta. De lo cual se desprende, que la actividad de investigación tiene ante sí el reto de analizar y aprehender las relaciones entre las fuerzas que generan el conflicto social, en un proceso de reproducción conceptual de lo real, a través de una reflexión respaldada con categorías que captan ésta realidad en su sentido histórico. La teoría crítica de la sociedad es la expresión del enfoque dialéctico contemporáneo y ha conducido a asumir una postura filosófica, que contrapone la sociología dialéctica a la sociología empírica. Como una derivación de su lógica interna, ha resultado que investigar es una acción consiente y de compromiso, por la cual el actor del proceso investigativo no puede ignorar que forma parte de una totalidad, en la cual debe desarrollar su capacidad crítica frente al orden social. El objetivo de la teoría crítica, más allá del ejercicio científico, es la transformación, de allí la diferencia con la sociología empírica analítica. Esta última asume el sentido positivista del concepto de sistema social y desde este enfoque interpreta a la realidad regulada por procedimientos normativos, tomados como requisitos funcionales, tales como equilibrio e integración de las leyes naturales.

\section{El paradigma cualitativo en la investigación social}

El hilo conductor del modelo cualitativo de la investigación, lo constituye el interés por entender la conducta de los actores, a través de la interpretación de las formas de vida que los caracteriza en la sociedad. Esto conlleva a la elabora- 
ción de constructos, a partir del sentido común de los actores en sus escenarios sociales. El llamado paradigma cualitativo busca la interpretación del accionar de la gente y lo relaciona con la conducta social que les es propia. La metodología cualitativa no tiene pretensiones de universalización y plantea la contextualización de la teoría en espacio y tiempo, sus enunciados son afirmaciones cuya validez se limita en el ámbito cultural e histórico. Por otro lado, rechaza la idea de cuantificar la realidad humana y rescata la importancia del "contexto, la función y el significado de los actores humanos" (Martínez, 1991:5). Trata de interpretar el accionar de la gente, es decir, sus motivos y creencias, que permiten desentrañar la naturaleza de la vida humana. Metodológicamente sus representantes se interesan en el enfoque interpretativo y fenomenológico para reforzar su posición antipositivista, y en ésta medida se centran en las manifestaciones de la razón humana para revalorizar el papel del sujeto en la construcción de la realidad social. Es decir, la mente humana es la que elabora percepciones para reconstruir el objeto de la realidad, dándole forma a la "materia amorfa" primaria que le proporcionan los sentidos. Para ello, utiliza categorías o formas propias, estructuradas con presupuestos o reglas generales, lenguaje, filosofía y estructura teórica: "Estas formas, es decir, lo que se percibe y su significado dependerá de nuestra formación previa, de nuestras expectativas teóricas actuales, de nuestras actitudes, creencias, necesidades, intereses miedos, ideales [...] Los objetos, los eventos, las situaciones no tienen un significado en sí mismo; más bien el significado se lo confiere el observador. De aquí, la necesidad de recoger los datos ubicados siempre en su contexto y la importancia de recurrir a una técnica hermenéutica para interpretarlos, ya que su significado permanecería oculto ante un análisis meramente positivista" (Martínez, 1991: 22). Desde el punto de vista investigativo la metodología cualitativa cuestiona la generalización sistemática y descontextualizada de los métodos cuantitativos y plantea los métodos cualitativos que enfatizan la vida cotidiana como objeto de estudio, en la cual incluyen el sentido del actor. Esto significa el rechazo a las concepciones naturalistas, donde lo humano y lo histórico está reducido al modelo de las ciencias naturales. Entre las vertientes metodológicas que derivan del paradigma cualitativo se encuentran; el interaccionismo simbólico, la etnometodología y la investigación acción.

El interaccionismo simbólico, expresa su interés en comprender las relaciones sociales del individuo en el grupo. Se señala a Herbert Blumer de haber sido el primero en iniciar la conceptualización del interaccionismo simbólico, como el estudio de los símbolos y las creencias que son manejados por un conjunto de individuos que, a su vez, conforman grupos sociales. Así Blumer (citado por Damiani, 1994:181) sostiene que en la perspectiva del interaccionismo simbólico: "Los seres humanos actúan frente a las cosas en base a los significados que estos poseen para ellos. El significado de las cosas surge de la interacción social de un individuo con otro. El significado es un producto social, es creado, no está intrínseco en las cosas. Los significados de las cosas son manipulados y modificados mediante un 
procedimiento interpretativo que los individuos utilizan para encararlas". De manera que ésta es una construcción intelectual del paradigma cualitativo, donde predomina la dimensión psicológica para el abordaje social de la realidad, a través de las interacciones entre las emociones y pensamientos de los individuos, por un lado, y el comportamiento social, por el otro. Es decir, para el interaccionismo simbólico, la realidad social obedece a una construcción del sujeto, utilizando sus símbolos sociales y culturales. Su preocupación central es el estudio de la relación entre el yo y la sociedad, la cual aborda como proceso de comunicaciones simbólicas entre los actores sociales, en este aspecto ha realizado relevantes aportes para la comprensión de la socialización, de la comunicación y de la acción.

El máximo exponente de la etnometodología Harold Garfinkel, planteó la crítica de la sociología imperante, considerándola como limitada para describir las conductas de la gente corriente. Garfinkel (citado por Rusque, 2001:80) señala que la etnometodología "trata sobre las actividades prácticas, las circunstancias prácticas y el razonamiento sociológico práctico como tema de estudio empírico. Concediéndole a las actividades banales de la vida cotidiana, la misma atención que se concede habitualmente a los acontecimientos extraordinarios ....el centro de su indagación es el proceso por el cual los actores sociales producen y sostienen un sentido de la estructura social en la que interaccionan".

En general, la etnometodología tiene como finalidad estudiar los métodos que utilizan los individuos para otorgar sentido a las actividades cotidianas.
Su interés cognoscitivo no está en los métodos de investigación propiamente tales, sino en el objeto de estudio directamente. De manera, que para su creador Garfinkel, la realidad de los hechos sociales es objetiva, en la medida que expresa la concertación de las actividades de la vida diaria, este es el fenómeno fundamental a ser estudiado por la etnometodología, la cual investiga como las personas construyen su mundo. Este enfoque considera la racionalidad de lo cotidiano como una cualidad que requiere ser investigada, con la finalidad de conocer como los actores participan en la sociedad, dándole sentido a las actividades que dirigen a los demás.

\section{La investigación-acción participante}

En esta modalidad nos referimos al esfuerzo sociológico latinoamericano de producir una alternativa metodológica, para fortalecer intelectualmente a las mayorías excluidas, por la tendencia monopolizante del conocimiento tradicional que limita cada vez más la participación en los espacios de toma de decisiones políticas, profundizando el sesgo ideológico hacia las estructuras de poder que marginan al sujeto histórico. De manera que la investigación-acción se plantea la necesidad de colocar el conocimiento transformador al servicio de los amplios sectores sociales. En este sentido, además de acciones de investigación incorpora acciones pedagógicas, dirigidas al colectivo que debe participar en la investigación, desapareciendo la separación del especialista con el investigador, ya que ambos son actores comprometidos 
con la acción social, en un ámbito espacial-temporal.

La direccionalidad de los investigadores es la producción de conocimientos para incorporar modificaciones intencionadas a la realidad de la que forman parte. Fals Borda (1987), propone la investigación participativa para producir un conocimiento que tenga validez para la práctica social y política de los actores sociales, ya que la crisis de las ciencias sociales tradicionales ha conllevado a un aislamiento de los intelectuales con respecto a las organizaciones de base, lo que ha evidenciado que ya no son los únicos capaces de transmitir conocimientos a las movimientos sociales y a los trabajadores. La investigación social como simple ejercicio intelectual ha perdido credibilidad. Desde esta perspectiva la investigación-acción implica la formación permanente de los sectores populares para que comprendan críticamente la realidad y colectivamente organicen la participación y dirección de los procesos de transformación. En virtud de esta connotación holística del proceso investigativo se replantea la relación sujeto-objeto y se reconoce que la ciencia debe adquirir una dimensión pública: "Con el concepto de compromiso se admite la esencia valorativa en la ciencia [...] un compromiso para la transformación, un compromiso en ese contexto de transformación una sociedad inadmisible" (Fals Borda, 1987:16).

\section{Lineamientos para la formación metodológica del gerente}

La preparación que se requiere para dirigir los sistemas productivos con una orientación social es el punto de partida, que asumimos, para la formación de los gerentes. De tal manera, que lo que algunos han dado en llamar la competitividad de la gerencia, incluye además de conocimientos técnicos, la posibilidad de transferir valor intelectual a las empresas, generando capacidad para la creatividad e innovación de las personas, y su disposición para incorporarse a las nuevas ideas de la transformación constante de la sociedad del conocimiento. No sólo se trata de mejorar la calidad de los productos y servicios con la incorporación de nuevas tecnologías, es prioritario un replanteo de los programas para preparar y formar a los gerentes y acelerar el cambio, para la creación de nuevas situaciones de justicia y equidad a nivel de la sociedad en circunstancias siempre complejas de la globalización: "Un cambio de tal complejidad es, aún más que el caso de la empresa, un cambio cultural. De allí lo doloroso, lo prolongado y lo difícil de la transformación" (Pérez, 1998:73).

Entre los requerimientos del nuevo esquema de formación gerencial, está el fortalecimiento de la capacidad de abstracción, a través del principio de concatenación universal, el cual promueve la visión de totalidad. Esto es posible si se refuerza la habilidad de la lectura critica y la profundización del lenguaje simbólico. Está por otra parte, la formación técnicocientífico para comprender la relación; ciencia, tecnología y sociedad, pues no sólo se trata de un alfabetismo científico, si no de apropiarse del estado del arte en el campo gerencial, para fortalecer la relación de las teorías con la realidad. Esto se resume en la búsqueda de una sólida formación de base que apoye la especia- 
lización polivalente y holística y que permita a los directivos participar en todos los niveles de la organización.

Otro de los componentes de la formación gerencial está relacionado con la capacidad para plantear y resolver problemas interesantes y relevantes. Se trata de salir del pensamiento único que nos viene de las modas gerenciales, esto se puede lograr a través de un ejercicio crítico y un pensamiento autónomo que se genera en relaciones colaborativas y en actividades como: Planificación concertada, trabajo en equipo, capacidad de diálogo, solución de conflictos, liderazgo efectivo, desarrollo de valores, capacidad de cambio. En estas actividades se requiere el entrenamiento metodológico para todos los miembros de las organizaciones, pues se trata de un proceso de aprendizaje colectivo con claridad de propósitos hacia el logro de la sensibilidad, la confianza y la flexibilidad entre quienes conviven en un determinado espacio laboral. Se trata en este sentido, de asumir la gerencia como un asunto colectivo y público para convertir la información técnica - administrativa en un conocimiento compartido, lo cual se ve favorecido por la proliferación de las actuales redes informáticas. Dicho de otra manera, se trata fundamentalmente de convertir la información en conocimiento, siendo el conocimiento la reflexión de la información (Savater, 1997).

De otra parte, la información gerencial se debe ampliar de acuerdo con la demanda ecológica mundial y en materia de preparación profesional se debe promover las estratégicas del desarrollo sustentable. Se requieren organizaciones autónomas y participativas, con visión am- bientalista, acerca de las demandas del entorno comunitario y del sector productivo laboral. Ello implica flexibilizar los programas para concienciar o humanizar a los lideres empresariales, pues se necesitan organizaciones accesibles e interactivas, conectadas en la autopista de la información de la red de redes. A su vez los cursos gerenciales deben discutir ampliamente los indicadores de pertinencia, calidad y equidad social, como asuntos comunes en los espacios públicos. Este actualizado filósofo de la educación como es Fernando Savater, ha rescatado el "valor de educar" como elemento central de nuestra civilización: "... el hecho de enseñar a nuestros semejantes y aprender de nuestros semejantes es mas importante para el establecimiento de nuestra humanidad que cualquiera de los conocimientos que así se perpetúan o transmiten" (Savater, 1997: 31).

Este programa de formación gerencial, se ha orientado por esta concepción del valor de educar, y ha promovido un conjunto de principios y atributos, entre los más importantes, se destacan: La objetividad que como aspiración postula una apropiación del objeto del conocimiento libre de sesgo y prejuicios. Pero al lado de la intersubjetividad, expresa el reconocimiento de las distintas posiciones $y$ valores que intervienen en nuestras prácticas y condicionan el proceso de formación. Otro principio es el de la heterogeneidad, el cual nos conduce a establecer la especificidad de cada proceso de formación gerencial. También está el principio de corresponsabilidad y la participación, relacionado con la conducta proactiva que debe promover el sistema de formación de los directivos. Es de vital 
importancia la visión holística e integradora de los componentes del proceso gerencial y su carácter multidisciplinario para entender la organizaciones como una totalidad compleja de múltiples determinaciones. También el enfoque estratégico que alude a la promoción de una visión compartida de la organización. El enfoque motivador resulta imprescindible para mantener la relación estrecha del nivel de satisfacción del desempeño del gerente, es decir la relación permanente entre la eficacia y la eficiencia.

\section{8. Éxito gerencial y compromiso social}

Algunas universidades han dedicado sus esfuerzos a la enseñanza gerencial, pero han tenido poca influencia, ya que solo hace pocos años que empezaron a ofrecer programas académicos vinculados con las áreas de recursos humanos, finanzas y producción. Vale decir, que en estos programas comúnmente los profesionales participan por iniciativa propia, ajenos a algún plan de carreras dirigidos por la empresa en la que laboran. El esfuerzo que realizan las instituciones académicas es poco significativo, ya que el desarrollo de la dirección empresarial ha recaído básicamente en la consultoría privada, la cual mas que una responsabilidad educativa formal, para promover un conocimiento científico, se auto impone la tarea de fabricar gerentes exitosos. Metafóricamente, se trata de una especie de parto gerencial, que es inducido por consultores, a través de la introyección de condiciones profesionales para crear una elite empresarial en el ejercicio actualizado de la dirección. Esta lógica edu- cativa se plantea lograr la efectividad del gerente en un escenario global de negocios sometidos a condiciones de competitividad cada vez mas elevadas.

La consolidación de la elite gerencial tiene su fundamento epistemológico en el modelo clásico de la burocracia de Weber. En este modelo se concreta la eficiencia para alcanzar los mas altos niveles de rentabilidad. Los atributos de la elite se refieren a un alto grado de especialización técnica, estructura jerárquica de la autoridad, cuerpo normativo para gobernar la organización, el liderazgo y conocimientos administrativos. En realidad este discurso de la burocracia predomina en la consultoría que se realiza en el núcleo empresarial privado, el interés está en la formación de capacidades y habilidades gerenciales a los fines de ejercer liderazgo, por ello se hace uso de la llamada teoría de la organización cuyos componentes principales son los siguientes: El comportamiento de los gerentes como estrato social de la elite, las relaciones de jerarquía de la dirección empresarial, la gerencia como ejercicio de control de la organización y la gerencia como proceso de conocimiento. Estos componentes, presentes en la consultoría empresarial, evidencian el carácter pragmático de la teoría de la organización, la cual surgió del análisis de la burocracia como matriz epistemológica, dedicada al estudio de las conductas de las personas en las empresas y los factores que afectan la estructura de las mismas, pero fundamentalmente su interés se centra en "The management of innovatión" que plantean Buns y Stalker (1961).

El éxito que busca la consultoría gerencial se concentra en la difusión de 
estilos donde predominan la orientación pragmática de los recursos humanos y la producción. Se limita a los aspectos operativos de la dirección de la empresa, es decir, a las capacidades y habilidades del gerente, acompasadas con una visión instrumental del conocimiento, asociada al uso exclusivo de las ventajas comparativas para el logro de la rentabilidad económica. No hay dudas que la orientación de esta consultoría se concreta a través de conocidas técnicas de simulación. Mediante esta simulación se transfieren procesos estandarizados de dirección y se adoptan estilos de liderazgo diseñados en función de una imagen empresarial de laboratorio y un modelo de clima organizacional preconcebido fuera de nuestra realidad. La concepción del capital intelectual es una de las expresiones mas palpables del pragmatismo gerencial, incorpora la lógica instrumental de la consultoría, para imponer las modas del entrenamiento, concebida para internalizar en el gerente el carácter marcadamente material de las relaciones laborales. Esta tesis, evidencia un sesgo economicista que ofrece una visión limitada y unilateral de la formación del gerente, a quien en lugar de dimensionar como el sujeto del proceso de dirección, con liderazgo en la sociedad, lo limita a ser objeto de las técnicas y modalidades gerenciales. En la medida en que se reduce la dirección empresarial a un problema operativo de la gerencia, su carácter social se pierde. Ello ocurre en la llamada gerencia del capital intelectual, cuando queda atrapada en las modas que circulan en el planeta ofreciendo soluciones a la rentabilidad como variable asociable a la calidad empresarial.
La superación de las tendencias gerenciales dominantes, exige el fortalecimiento de los programas de formación metodológica, que motoricen el proceso de cambio hacia la sociedad sustentable del desarrollo humano, donde recobremos nuestras raíces históricas, nuestra herencia independentista y reivindiquemos en ellas la unidad del pensamiento latinoamericano. Más aun, los metodólogos estamos obligados a enfrentar el pensamiento único neoliberal, y denunciar los extravíos de su modelo desnacionalizador, cuyos resabios pretenden convertirse en un muro de contención para la enseñanza crítica de los gerentes. El programa para la enseñanza metodológica de los gerentes, exige el abordaje psicosocial de la gerencia concebida como un hecho complejo, expresión de la multiplicidad de disciplinas que involucra. En esta perspectiva, la formación gerencial tiene, por un lado, la obligación de profundizar el conocimiento de la situación actual de la sociedad, y por el otro, estimular el talento y la inteligencia de los gerentes, a través de un Proyecto Político-Académico que los coloque en la búsqueda de la excelencia. Sin embargo hay que advertir que esta excelencia no surge espontáneamente, ni se decreta burocráticamente. Hay que construirla mediante un ejercicio creativo, integrador y trascendente, donde estén presentes los indicadores de la calidad técnica científica y la equidad social.

Las expectativas de cambio del paradigma gerencial encuentra en los programas de formación metodológica su motor principal, pues se trata de la transformación de las maneras de pensar, actuar y organizar de los directivos empre- 
sariales. Se plantea construir un sistema con estrategias métodos y herramientas para crear organizaciones que asuman la complejidad y la incertidumbre que genera el cambio como condición recurrente de la sociedad. De ello se deriva la necesidad de formular un paradigma sistémico que supere la previsibilidad del modelo burocrático: "El modelo burocrático de Weber parte de la suposición de que el comportamiento de los miembros de la organización es perfectamente previsible: todos los funcionarios deberán comportarse de acuerdo a las normas y reglamentos de la organización con el fin de que ésta alcance la máxima eficiencia posible. En la burocracia todo se establece con el fin de prever anticipadamente todas las actuaciones y rutinizar su ejecución, para que la máxima eficiencia del sistema se alcance plenamente" (Chiavenato; 1993).

El aludido carácter sistémico se considera una condición indispensable para el ejercicio de una nueva dirección empresarial donde confluya la misión, con la estructura, con el funcionamiento, con los procesos y con las oportunidades $y$ amenazas que se encuentran en el entorno. Conviene señalar que el programa de formación metodológica para gerentes se basa en el enfoque psicosocial, que encuentra entre sus fundamentos epistemológicos el planteamiento de Maritza Montero (1997: Contraportada), acerca de la ideología, alineación e identidad nacional: "El fenómeno de la dependencia es tanto externo cuanto interno. A los factores socioeconómicos y culturales se unen factores psicológicos, que se expresan en una conducta particular, reproductora de las circunstancias que dan lu- gar a ella y que se traduce en una representación social caracterizada por estereotipos negativos y una imagen nacional devaluada". Siguiendo este enfoque psicosocial, convendríamos en afirmar que el uso circunstancial de la consultoría externalizada ha conllevado, por sus intereses foráneos, a un estado de alienación, que negando la autoimagen ha reforzado la tendencia a la estereotipación de las organizaciones empresariales de América Latina.

En los últimos años los expertos, gurúes del argot gerencial, han venido realizando esfuerzos para estandarizar los procesos, los medios y los resultados de la gerencia, de acuerdo con la lógica globalizadora que impulsan la mayoría de los consultores de orientación neoliberal. En esencia quienes han estado al frente de éstas instituciones de formación gerencial no se han planteado con claridad el diseño de una estrategia formativa, donde se relacionen los factores socioeconómicos, con los factores culturales, y con los factores psicológicos. El problema de la identidad prácticamente lo han catalogado como un asunto folklórico de las prácticas directivas que ellos consideran ya superadas, esto refleja el predominio de una especie de etnocentrismo gerencial, que subyace en la era de la globalización, que tiende a desdeñar el carácter científico de la gerencia local.

El programa de enseñanza metodológica para gerentes plantea que los metodólogos, debemos asumir una posición de compromiso con el proyecto científico nacional (Varsavsky, 1975), que contenga los siguientes elementos: Desempeño creativo y eficiente para mejorar la relación de la gerencia con el entor- 
no; formación y reconversión profesional permanente; convivencia comunitaria a través de un nuevo lenguaje para el dialogo y la reflexión, donde impere la argumentación, la negociación, el consenso y la integración en función del colectivo; comunicación racional y espiritual que articule la práctica y la teoría y que permita que de la palabra siempre se pase a la acción; conocimiento científico-tecnológico convertidos en componentes impulsores de la redención social; inteligencia artificial para integrar al hombre con la técnica en función de la calidad de vida; formación para la vida que proyecte la felicidad espiritual centrada en los valores de la solidaridad y respeto opuesto a la alienación de la llamada industria cultural, cuya finalidad es estimular el consumismo. Los compromisos de la gerencia conllevan a la promoción de la conciencia colectiva para enfrentar con fuerza las tendencias alienantes $y$ fortalecer el sentido de pertenencia, la identidad con la patria y elevación de la autoestima de los investigadores para luchar por una gerencia que promueva la equidad y la pertinencia social.

\section{Conclusiones}

La formación metodológica gerencial, basada en la tesis de la diversidad metodológica, se nos presenta como una opción flexible capaz de superar la visión estrecha del modelo empírico-analítico, que a través de un instrumentalismo de técnicas y procedimientos soslaya la reflexión teórica acerca del objeto de estu- dio. Dentro de la opción epistemológica asumida, la actividad científica aparece como una acción determinada por condiciones históricas y sociales. De allí se deriva el alcance de esta propuesta de formación metodológica, relacionado con la adquisición de la responsabilidad social del gerente, a través del estudio crítico de los modelos cualitativos y cuantitativos, para lograr los objetivos de mantener el rigor científico de la práctica investigativa, contextualizar el conocimiento, recrear la acción gerencial, y procurar una gestión directiva de excelencia. La metodología utilizada para el desarrollo del Programa se basó en una dinámica sistémica del proceso de aprendizaje-enseñanza, con una entrada con insumos teórico-metodológicos, en un proceso donde se realizó el trabajo individual del estudiante y en un producto donde se concretaron los resultados de la investigación.

Finalmente, en este trabajo se ha demostrado que la formación metodológica con tal orientación, le permite al gerente alcanzar las siguientes competencias: Visión de totalidad, dominio del estado del arte en el campo de conocimiento que aborda, capacidad para vincular la ciencia y la tecnología con la sociedad, habilidad para comunicar los impactos de la ciencia, disposición para asumir la investigación como un asunto de utilidad pública, capacidad para trabajar en redes y convertir la información en conocimiento y disposición para mantener la autonomía, la confianza y la sensibilidad intelectual. 


\section{Bibliografía Citada}

Bachellard, Gaston (1976), "La formación del espíritu científico". Buenos Aires. Siglo veintuno editores, S.A.

Buns, T. y Starker, G.M. (1961), "The management of innovation". Londres, Editorial Tavistock.

Chiavenato, Idalberto (1993), “Administración de Recursos Humanos". Colombia, Editorial MC Graw Hill.

Damiani, Luis (1994), “La diversidad metodológica en la sociología". Caracas, Fondo Editorial Tropykos.

Fals Borda, Orlando (1987), "La investigación participativa" Montevideo, Editado por Banda Oriental.

Kuhn, Tomas (1981), "La estructura de las revoluciones científicas". México. Editorial Fondo de cultura económica.

Martinez, Miguel (1991), “La investigación cualitativa etnográfica en educación". Caracas. Editorial texto.

Marx, Karl (1979), "Miseria de la Filosofía". Madrid. Editorial Aguilar.

Marx, Karl y Engels Olson (1974), "El manifiesto Comunista". Madrid. Editorial Ayuso.
Montero, Maritza (1997), "Ideología, alienación e identidad nacional". Caracas, Ediciones UCV.

Perez, Carlota (1998), "Desafíos sociales y políticos del cambio del cambio de paradigma tecnológico". Caracas, Editado por UCAB.

Poulantzas, Nicos (1977), "Poder político y clases sociales en el estado capitalista". México, Siglo veintiuno editores S.A.

Romero, Juan (2000), "La formación de los cuadros directivos en la perspectiva CTS" Ciudad Guayana. Material mimeografiado.

Rusque, Ana María (2001), "De la diversidad a la unidad en la investigación cualitativa". Caracas. Editorial Faces UCV.

Savater, Fernando (1997), "El valor de educar”. Colombia, Editorial Ariel.

Varsavsky, Oscar (1975), "Ciencia, Política y Cientificismo". Buenos Aires. Centro editor de América Latina.

Weber, Max (1973), "Ensayos sobre metodología sociológica". Buenos Aires, Amorrortu editores. 